\title{
Coherent Control of Angular Momentum Transfer in Resonant Two-Photon Light-Matter Interaction
}

\author{
D. A. Malik, A. V. Kimel, A. Kirilyuk, Th. Rasing, and W. J. van der Zande* \\ Radboud University Nijmegen, Institute for Molecules and Materials, P.O. Box 9010, NL-6500 GL Nijmegen, The Netherlands
}

(Received 17 December 2009; published 29 March 2010)

\begin{abstract}
We show experimentally and theoretically that a polarization-shaped femtosecond laser pulse with a zero net angular momentum creates a net angular momentum in atomic rubidium during resonant twophoton excitation. The necessary conditions for the creation of a nonzero angular momentum as well as the excitation efficiencies are analyzed in the framework of second-order time-dependent perturbation theory.
\end{abstract}

DOI: 10.1103/PhysRevLett.104.133001

With the advent of femtosecond lasers coherent control of light-matter interactions has become an important area of research of broad interest in both physics and chemistry $[1,2]$. This is also true for magnetism, where laser control of magnetic order has become a subject of intense research for the last decade [3]. Ultrafast demagnetization of a magnetic material and even magnetization reversal has been demonstrated using ultrafast optical radiation [4,5]. Manipulation of magnetic order requires angular momentum transfer. It has been reported that excitation of a medium with a femtosecond laser pulse results in a large change of angular momentum in magnetic subsystems at the surprisingly fast time scale of the laser pulse. This time scale is much shorter than any known relaxation processes in solids. Therefore, it was suggested that the electromagnetic field directly controls the necessary angular momentum transfer [6,7].

Normally, in an electric dipole transition, circularly polarized photons are required to change the magnetic quantum number of an electron. In the presence of spinorbit interaction a one-photon process probes the magnetization of a medium via the magneto-optical Faraday and Kerr effects [8]. The combination of spin-orbit interaction and two-photon stimulated Raman scattering provides a plausible mechanism for femtosecond control of spins [9,10]. However, a detailed understanding of the magneto-optical effects with laser pulses shorter than the time of optical decoherence is still lacking $[11,12]$. The problem is further complicated in solids, where the presence of translational symmetry makes that angular momentum is not a good quantum number and that optical selection rules are not rigorous. However, even for an ideal system the role of optical coherence in the transfer of angular momentum during a two-photon interaction has not been fully understood yet.

The problem of angular momentum transfer during the off-resonant interaction of light with a simple three-level system was considered by Dudovich et al. [13]. However, no possibility of creating net angular momentum in the final state by a laser pulse with linearly polarized frequencies was considered. In this Letter we investigate two-
PACS numbers: 32.80.Qk, 32.80.Wr, 42.65.Re, 75.78.Jp

photon excitation of rubidium atoms to individual magnetic sublevels, both resonantly and off resonantly. While using linearly polarized shaped laser pulses, we show that the coherence between the linearly polarized waves at different frequencies leads to the creation of net angular momentum. We also establish the crucial role of the interference between resonant and off-resonant excitation in the transfer of angular momentum.

Rubidium is a favorable candidate for coherent control studies using standard femtosecond Ti:sapphire laser systems. It has two resonant transitions at $\lambda_{i g}=780.2$ and $\lambda_{f i}=776.0 \mathrm{~nm}$ (corresponding to $1.59 \mathrm{eV}$ and $1.60 \mathrm{eV}$, respectively) both within the spectral band of a 75 femtosecond laser pulse [see Fig. 1(a)]. Via a two-photon process the final $5 d$ state is excited, while its population and angular momentum are determined by observing fluorescence. The atoms decay spontaneously to the ground state via the $6 p$ level, where we observe the $6 p$ to $5 s$ transition at
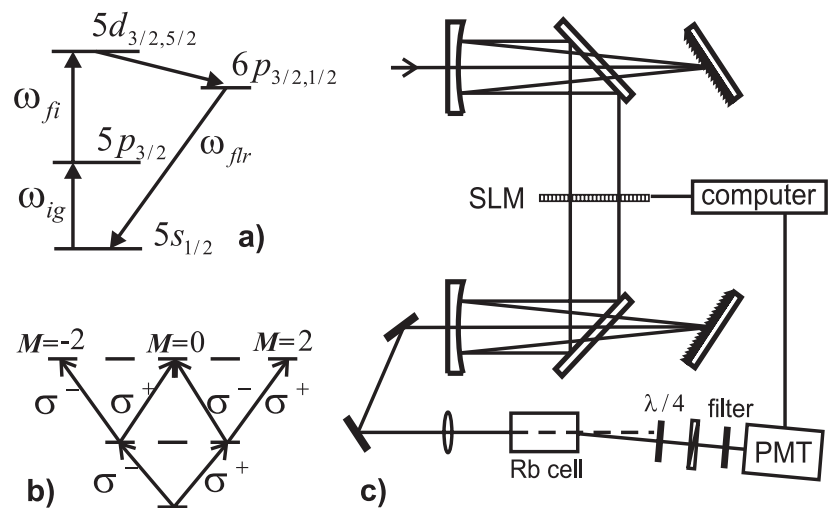

b)

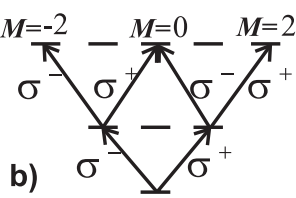

c)

FIG. 1. (a) The level scheme of rubidium. The spontaneous emission from $6 p$ to $5 s$ state at $420 \mathrm{~nm}$ is measured. (b) The excitation scheme, including $m$ sublevels. The linear polarization is a superposition of circularly polarized components, and quantization axis is the $k$ vector of the laser light. (c) Scheme of the experimental setup. The pulse shaper has a $4 f$ geometry. The mirror after the shaper is set close to Brewster's angle. The fluorescence detection system has a $\lambda / 4$ plate, a polarizer, and a PMT with attached narrow-band filter. 
$420 \mathrm{~nm}$. The nonamplified laser pulses have a bandwidth of $12 \mathrm{~nm}$ (corresponding to $75 \mathrm{fsec}$ transform-limited pulses) centered at a wavelength of $778.1 \mathrm{~nm}$. The pulse shaper [see Fig. 1(c)] has a $4 f$ geometry, as in Präkelt et al. [14]. Details of the shaper can be found in [15]. In the present experiment we rotated the polarization of part of the spectrum by $90^{\circ}$ and scanned the position of the step between the orthogonally polarized parts. In some experiments, part of the spectrum was blocked in the Fourier plane and phase step was also applied. Care has been taken to ensure that the relative intensities of the spectral components are not affected by the polarization shaping.

Rubidium vapor was contained in a cell at room temperature. The angular momentum of the final $5 d$ level was deduced from the fluorescence observed at a small angle relatively to the laser beam propagation direction. The main observable was the intensity contrast between leftand right-handed polarized fluorescence. The detection system consisted of a quarter-wave plate, a polarizer, and a photomultiplier tube (PMT) with attached narrow-band filter around $422 \mathrm{~nm}$. Two effects determined the maximum observable contrast. First, due to the lifetime of the $5 d$ and the $6 p$ states, fine structure and hyperfine structure coupling reduced the alignment, created immediately after the excitation pulse. Second, we detected the second photon in the cascaded spontaneous fluorescence decay which further reduced the contrast. From calibration measurements, when exciting rubidium with purely circularly polarized light, the maximum observable contrast in the fluorescence was determined to be about $55 \%$.

The results of an experiment as a function of the spectral position of the change in polarization are shown in Fig. 2(b). We show both the total fluorescence intensity, representing the total $5 d$ population, and the amount of left- and right-handed circularly polarized fluorescence. The experimental data reveal two important features: the enhancement of the fluorescence intensity around resonances and the strong contrast in the fluorescence of opposite helicities, implying that the atoms have acquired a net polarization.

To understand this effect we performed calculations in the frame of second-order time-dependent perturbation theory. We restrict the treatment to orbital angular momentum only. This restriction preserves the essence of the experiment. As quantization axis the propagation direction of the light is chosen, allowing for a consistent choice for the angular momentum wave functions. The two linear polarizations are represented as a superposition of leftand right-handed circularly polarized light:

$$
\vec{E}_{x}=\vec{\sigma}^{+}+\vec{\sigma}^{-}, \quad \vec{E}_{y}=i \vec{\sigma}^{+}-i \vec{\sigma}^{-},
$$

where $\vec{E}_{x}$ and $\vec{E}_{y}$ are orthogonal, linearly polarized spectral components, $\vec{\sigma}^{+}$and $\vec{\sigma}^{-}$are right- and left-handed circularly polarized components, and $\left|\vec{\sigma}^{+}\right|=\left|\vec{\sigma}^{-}\right|=\sigma^{+}=$ $\sigma^{-}$.
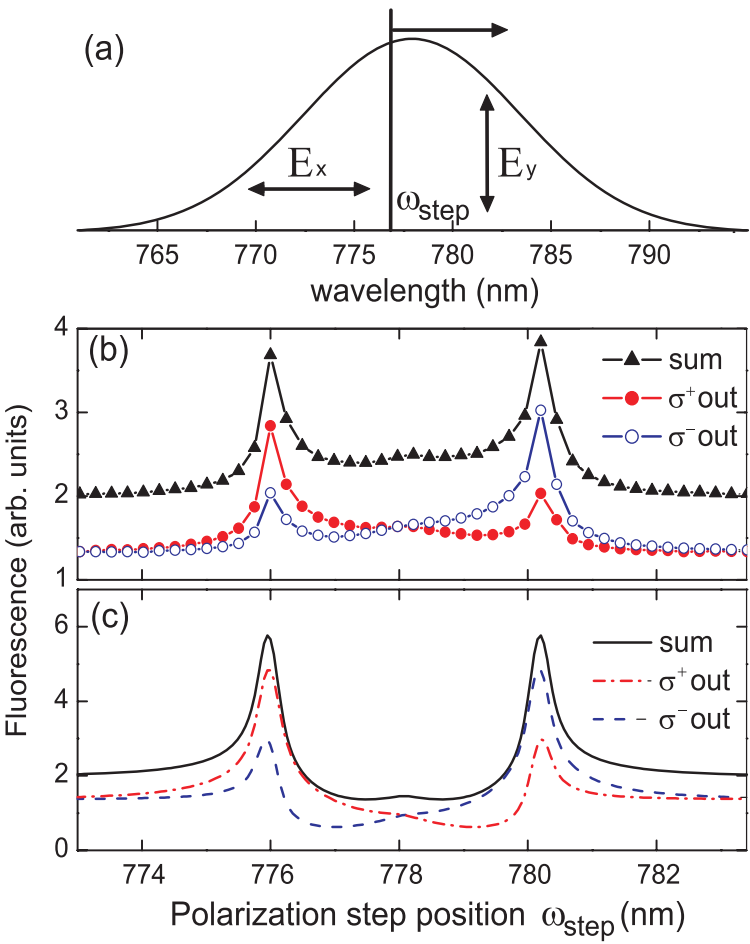

FIG. 2 (color online). (a) Scheme of the change in polarization in the spectrum. In experiment $\omega_{\text {step }}$ is scanned. (b) Observed total fluorescence intensity and circular polarization contrast indicate induced angular momentum. (c) The calculations, taking into account spectral resolution of the shaper and experimentally verified reduction in polarization contrast.

Figure 1(b) shows the excitation scheme of the magnetic sublevels in this reference frame. In the perturbative limit, the final $5 d \mathrm{~m}$-states amplitudes involving an intermediate resonant level are given by [16]:

$$
\begin{aligned}
a_{f, m} \approx & -\frac{1}{i \hbar^{2}} \mu_{f i} \mu_{i g}\left[i \pi E\left(\omega_{i g}\right) E\left(\omega_{f i}\right)\right. \\
& \left.+\wp \int_{-\infty}^{\infty} \frac{E(\omega) E\left(\omega_{f g}-\omega\right)}{\omega-\omega_{i g}} d \omega\right],
\end{aligned}
$$

where the sublevel index " $m$ " runs from -2 to 2 , and where $\omega_{i g}$ and $\omega_{f i}$ are the resonant frequencies between the intermediate and ground and the final-intermediate levels, respectively, $\mu_{i g}$ and $\mu_{f i}$ are the $m$-dependent transition dipole moment matrix elements, and $\wp$ is the principal value of Cauchy. Equation (2) contains a resonant and an off-resonant term. The resonant contribution depends only on the spectral components at resonant frequencies and is $\pi / 2$ shifted from the term containing the integration over all remaining nonresonant photon pairs in the pulse.

The different $m$ levels react on different combinations of left-handed and right-handed circularly polarized light [see Fig. 1(b)]. Depending on the position of the step between the orthogonally polarized parts, the two excitation photons either both are linearly polarized along the $x$ direction, 
TABLE I. Electric field combinations contributing to the final state amplitudes.

\begin{tabular}{lccc}
\hline \hline Contribution to & Both $x$ polarized & Both $y$ polarized & Both orthogonal \\
\hline$m=2$ & $\sigma^{+}(\omega) \sigma^{+}\left(\omega_{f g}-\omega\right)$ & $-\sigma^{+}(\omega) \sigma^{+}\left(\omega_{f g}-\omega\right)$ & $i \sigma^{+}(\omega) \sigma^{+}\left(\omega_{f g}-\omega\right)$ \\
$m=0$ & $2 \sigma^{-}(\omega) \sigma^{+}\left(\omega_{f g}-\omega\right)$ & $2 \sigma^{-}(\omega) \sigma^{+}\left(\omega_{f g}-\omega\right)$ & 0 \\
$m=-2$ & $\sigma^{-}(\omega) \sigma^{-}\left(\omega_{f g}-\omega\right)$ & $-\sigma^{-}(\omega) \sigma^{-}\left(\omega_{f g}-\omega\right)$ & $-i \sigma^{-}(\omega) \sigma^{-}\left(\omega_{f g}-\omega\right)$ \\
\hline \hline
\end{tabular}

or are both polarized along the $y$ direction, or the excitation involves a photon of each polarization. In Table I the combinations contributing to the final state amplitudes are given.

The only difference in the contributions to the $m=2$ and $m=-2$ levels is the sign change in the imaginary terms for photons with orthogonal polarizations. This gives the condition that for creation of angular momentum the final transition amplitude $a_{f}$ [as in Eq. (2)] should contain both real and imaginary terms. For laser pulses that are only shaped by a change in polarization, the $\pi / 2$ phase difference between the resonant and off-resonant contributions in Eq. (2) provides the necessary condition for creation of angular momentum in the final state, as is observed in experiment. This observation stresses the role of the interference between resonant and off-resonant excitation in the transfer of angular momentum.

The enhancement of the excitation efficiency near the resonant frequencies can be explained in terms of interference. Rotation of the polarization in the spectrum acts as a control parameter of the interference between excitation events to a certain $5 d m$ state by different spectral combinations, as expressed in Table I. Because of the singularity in the off-resonant term in Eq. (2) this has a particularly strong effect on the excitation efficiency at resonances. Another intriguing form of control of the interference by using phase shaping, as well as the role of the singularity in the off-resonant contribution was shown by Dudovich et al. [16].

The results of the calculation based on Eq. (2) and Table I are shown in Fig. 2(c). Apart from the absolute values of the enhancement at the resonances and the intensity when changing the polarization in between the resonances, qualitatively all features are reproduced. Our calculations take into account the finite spectral resolution of the pulse shaper and the experimentally verified reduction in contrast. Remaining discrepancies between observations and calculations are ascribed to spectral distortions caused in the shaper. The sudden phase jumps at the edges of the SLM pixels have a strong effect on the far-field spectral distribution [17].

To further corroborate the role of the resonant excitation, we have blocked part of the spectrum, including the resonant component at $776 \mathrm{~nm}\left(\omega_{f i}\right)$. Now our results showed no visible difference in the intensities of the fluorescence for opposite helicities (see Fig. 3).

We have shown that the interference between resonant and off-resonant excitation plays a crucial role in the creation of angular momentum in the final state. This condition might be generalized in a form that $a_{f}$ being complex is a sufficient condition for the creation of angular momentum. We have made $a_{f}$ complex, still blocking the resonant contribution, by adding a $\pi / 2$ phase shift at the position of the polarization step. Indeed, the fluorescence intensities of opposite helicities become unequal; the atoms gain a net polarization (see Fig. 3).

So far we have analyzed the angular momentum properties of the final state during a two-photon excitation in frequency space in terms of the interference phenomena. Analysis of the excitation in time reveals the correlation between the time structure of the pulse and the time behavior of both $5 p$ and $5 d$ states. We have performed time-dependent calculations by solving the Schrödinger equation in the perturbative limit, as in [15]. Figure 4(a) shows the electric field of the central part of the laser pulse when the step in polarization is positioned at the resonance

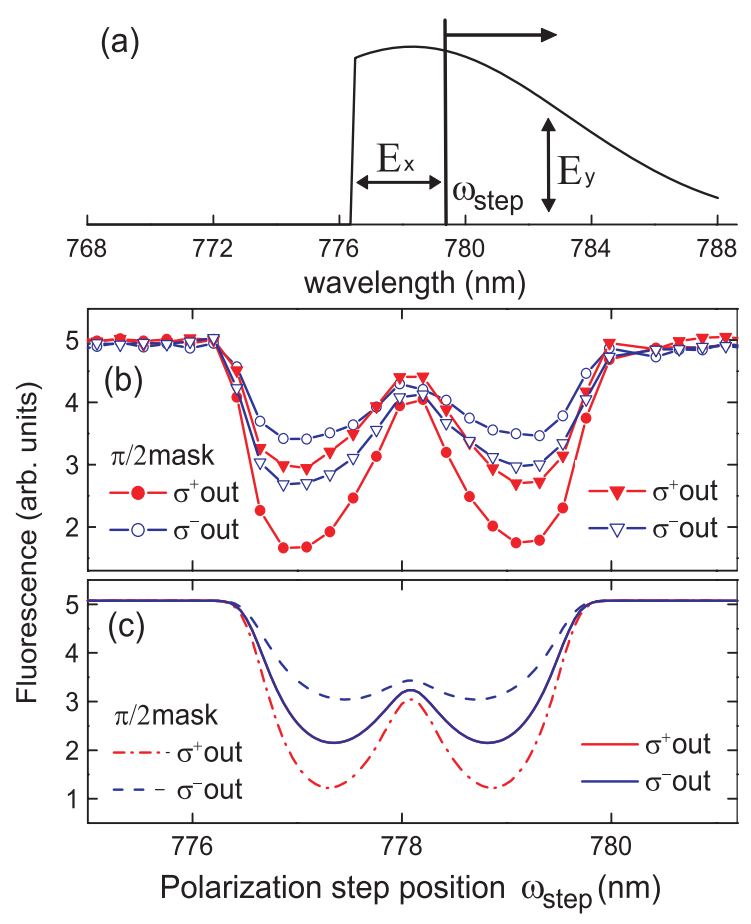

FIG. 3 (color online). (a) Scheme of the change in polarization in the spectrum. The resonant frequency at $776 \mathrm{~nm}\left(\omega_{f i}\right)$ is blocked. In experiment $\omega_{\text {step }}$ is scanned. (b) Observed circular polarization contrast. An extra $\pi / 2$ phase shift between orthogonal spectrum parts restores polarization contrast, inducing angular momentum. (c) The calculations, taking into account the spectral resolution of the shaper and experimentally verified reduction in polarization contrast. 

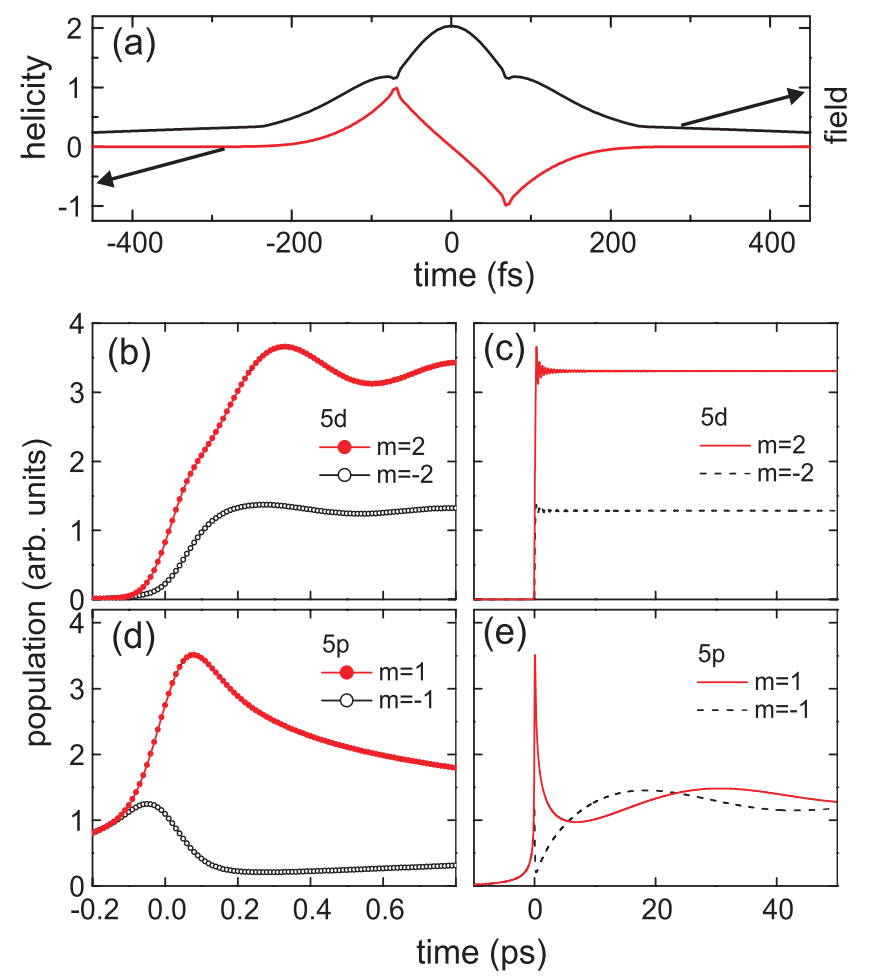

FIG. 4 (color online). (a) Electric field envelope and helicity $\vec{h}=i\left[\vec{E} \times \vec{E}^{*}\right] / E^{2}$ of the central part of the excitation pulse for polarization step at $780.2 \mathrm{~nm}\left(\omega_{i g}\right)$. (b) and (d) Population dynamics of the $5 \mathrm{~d} m= \pm 2$ states and $5 \mathrm{p} m= \pm 1$ states follow dynamics of the helicity during the pulse. (c) and (e) Angular momentum survives on a long time scale in the $5 d$ level and disappears asymptotically in the $5 p$ level.

at $780.2 \mathrm{~nm}\left(\omega_{i g}\right)$. The tails of the pulse are linearly polarized, while in the central part circular polarization occurs first in one and then in the other direction. In Fig. 4(b) populations of the $5 d m=2$ and $m=-2$ sublevels are plotted as a function of time during the first picoseond of the shaped laser pulse. The time delay between the growth of the $m=2$ and $m=-2$ populations reflects the change in polarization of the laser pulse. In Fig. 4(c) it is shown that the difference in dynamics survives on a long time scale.

The $5 p$ level is populated in a resonant one-photon process. Still, even here a strong difference in the angular state is present during the action of the laser pulse. In Fig. 4(d) the evolution of the populations of the $5 \mathrm{p} \mathrm{m}=1$ and $m=-1$ sublevels is shown. The step in polarization is located at the resonance at $780.2 \mathrm{~nm}\left(\omega_{i g}\right)$. The time structure of the pulse manifests itself as an enhancement of the excitation to the $m=1$ level, forming at the same time a stimulus to deexcite the $m=-1$ level. When the helicity reverses during the fast pulse the dynamics is reversed. Of course, during the ultrafast pulse the system cannot distinguish between different frequencies; instead the system follows the time evolution of the electric field in the pulse. Asymptotically, only the resonant component plays a role and the net angular momentum at the $5 p$ level disappears after a time of about 50 psec [see Fig. 4(e)]. The slow oscillation of the populations is due to the slight offset of the position of the polarization step from the exact resonance.

Clearly, angular momentum is exchanged between the rubidium atoms and the light in a complex time-dependent way. We note that the fact that during ultrafast pulse angular momentum can be exchanged in a nonintuitive fashion, such that the polarization of emitted light is altered, has been pointed out recently in an $a b$ initio study of laser-induced spin dynamics in $\mathrm{NiO}$ by Lefkidis et al. [18].

In conclusion, we have demonstrated coherent control of angular momentum transfer into the rubidium $5 d$ level in a two-photon excitation. We have found that even in the case of only linearly polarized spectral components, the $\mathrm{Rb}$ atoms obtain a net angular momentum. Necessary conditions for creating this net angular momentum are the multiphoton character and the existence of interference between resonant and off-resonant excitation: generally the amplitude $a_{f}$ [as in Eq. (2)] needs to be complex. Our results also reveal that during the interaction large amounts of transient angular momentum can be observed even in a one-photon transition. Our results provide important insights for understanding of the problem of angular momentum transfer from light to matter, opening potentially new perspectives for coherent control of a magnetic state of a medium.

The authors acknowledge the technical support of T. Toonen, A. van Etteger, and the financial support of FOM and NWO.

*w.vanderzande@science.ru.nl

[1] D. Meshulach and Y. Silberberg, Nature (London) 396, 239 (1998).

[2] Z. Chen et al., Chem. Phys. Lett. 198, 498 (1992).

[3] J. Stohr and H. C. Siegmann, Magnetism: From Fundamentals to Nanoscale Dynamics (Springer, Berlin, 2006).

[4] E. Beaurepaire et al., Phys. Rev. Lett. 76, 4250 (1996).

[5] C. D. Stanciu et al., Phys. Rev. Lett. 99, 217204 (2007).

[6] G. P. Zhang and W. Hubner, Phys. Rev. Lett. 85, 3025 (2000).

[7] J.-Y. Bigot et al., Nature Phys. 5, 515 (2009).

[8] A. K. Zvezdin and V.A. Kotov, Modern Magnetooptics and Magnetooptical Materials (IoP, Bristol, 1997).

[9] A. V. Kimel, A. Kirilyuk, and Th. Rasing, Laser Photon. Rev. 1, 275 (2007).

[10] A. V. Kimel et al., Nature (London) 435, 655 (2005).

[11] G. P. Zhang, Phys. Rev. Lett. 101, 187203 (2008).

[12] G. P. Zhang et al., Nature Phys. 5, 499 (2009).

[13] N. Dudovich, D. Oron, and Y. Silberberg, Phys. Rev. Lett. 92, 103003 (2004).

[14] A. Präkelt et al., Rev. Sci. Instrum. 74, 4950 (2003).

[15] A. S. Meijer et al., Phys. Rev. A 78, 053403 (2008).

[16] N. Dudovich et al., Phys. Rev. Lett. 86, 47 (2001).

[17] B. J. Sussman, R. Lausten, and A. Stolow, Phys. Rev. A 77, 043416 (2008).

[18] G. Lefkidis, G. P. Zhang, and W. Hubner, Phys. Rev. Lett. 103, 217401 (2009). 